\title{
Inferior Hip Dislocation Without Any Fracture: A Case Report
}

ISSN: 2576-8875

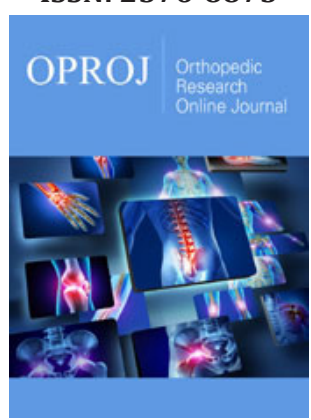

*Corresponding author: Seyyed Hossein Shafiei, Department of orthopaedic surgery, Tehran university of medical sciences, Tehran, Iran

Submission: 麅 November 18, 2020

Published: 監 February 22, 2021

Volume 8 - Issue 1

How to cite this article: Seyyed Hossein Shafiei, Salar Baghbani, Babak Siavashi, Farhad Mahdavi and Mohammad Reza Golbakhsh. Inferior Hip Dislocation Without Any Fracture: A Case Report. Ortho Res Online J. 8(1). OPROJ. 000676. 2021. DOI: 10.31031/OPROJ.2021.08.000676

Copyright@: Seyyed Hossein Shafiei, This article is distributed under the terms of the Creative Commons Attribution 4.0 International License, which permits unrestricted use and redistribution provided that the original author and source are credited.

\section{Seyyed Hossein Shafiei*, Salar Baghbani, Babak Siavashi, Farhad Mahdavi and Mohammad Reza Golbakhsh}

Department of orthopaedic surgery, Tehran university of medical sciences, Iran

\begin{abstract}
Background: Hip joint is inherently stable via strong ligamentous and bony structures and a high energy trauma is necessary to yield dislocation. Inferior hip dislocation is an epidemiologically and logically rare condition and to our knowledge only 12 cases have been reported in the English literature. The injury mechanism is poorly understood but generally is caused by a high energy trauma while hip joint is in an abducted position.
\end{abstract}

Case: In this article we are about to share a patient with inferior hip dislocation reduced by simple Allis maneuver under intravenous sedation in the emergency room facing no other complications after 12 months' follow-up

\section{Introduction}

hip joint stability is guaranteed by strong ligamentous and bony architecture surrounding the joint. Joint dislocation needs a high energy trauma in a particular direction to overcome the mentioned structures [1].

Posterior hip dislocation is the most common type of hip dislocation. Inferior hip dislocation is least common and consists of about 10 percent of all hip dislocations [2]. This subgroup is categorized by the mechanism of injury to more prevalent ischial type and the rarer obturator type or also known as the true inferior type [3]. To our best knowledge, there are only 12 case reports of inferior hip dislocation in the English literature so far [4-7]. Most of the mentioned cases are children which is believed to be an independent risk factor due to ligamentous laxity for all anterior hip dislocations. High energy trauma is obligated to cause this type of injury and position of the hip and knee joints during trauma dictates the type of dislocation [8].

Hip dislocation is an emergency and needs to be reduced within 6 hours to reduce the risk of future avascular necrosis of the femoral head [9] which in our case patient was reduced within 4 hours of the trauma in the operating room under IV sedation by Allis' maneuver. Patient started walking toe touch weight bearing the next day after the trauma and faced no complication in the 12 months follow-ups.

\section{Case Report}

A 42-year-old male was brought to our emergency room by Emergency Medicine Service (EMS) while the left lower limb was held at flexion and external rotation. Driving on a motorcycle (hip at flexion and external rotation and abduction) he was hit by a car from the right side and fell on his left hip. On arrival patient's vital signs were measured stable and neurovascular examination in lower extremity was intact. he was complaining of extreme pain on his left hip (while holding his left lower limb in an externally rotated position and claimed to be unable to lift the injured limb). On the portable anteroposterior (AP) X-ray (Figure 1) left hip dislocation was detected. Closed reduction (inline traction followed by rotation) under intravenous (IV) sedation was done. After reduction neurovascular examination was intact. 


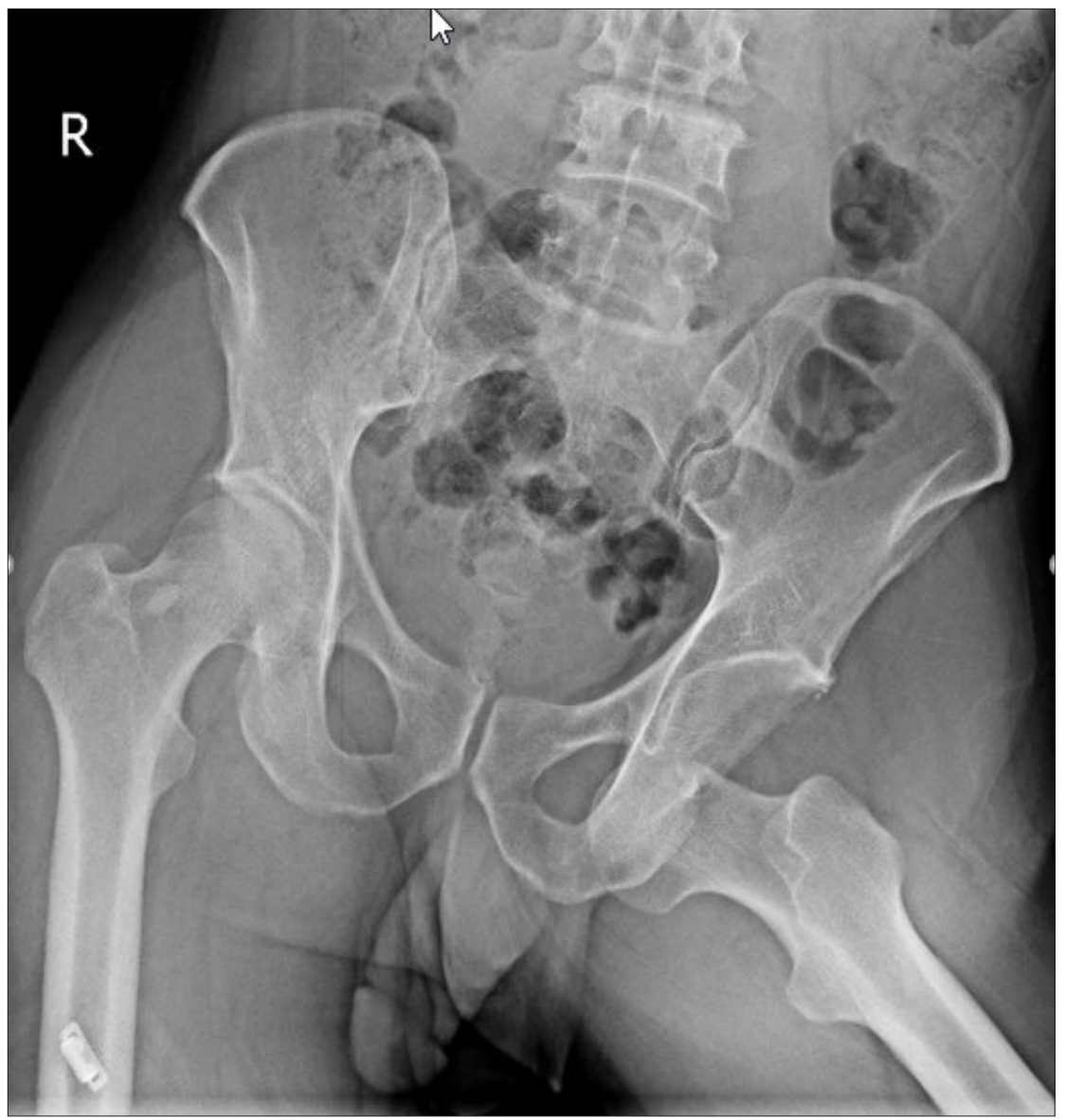

Figure 1: AP pelvic X-ray pre reduction.

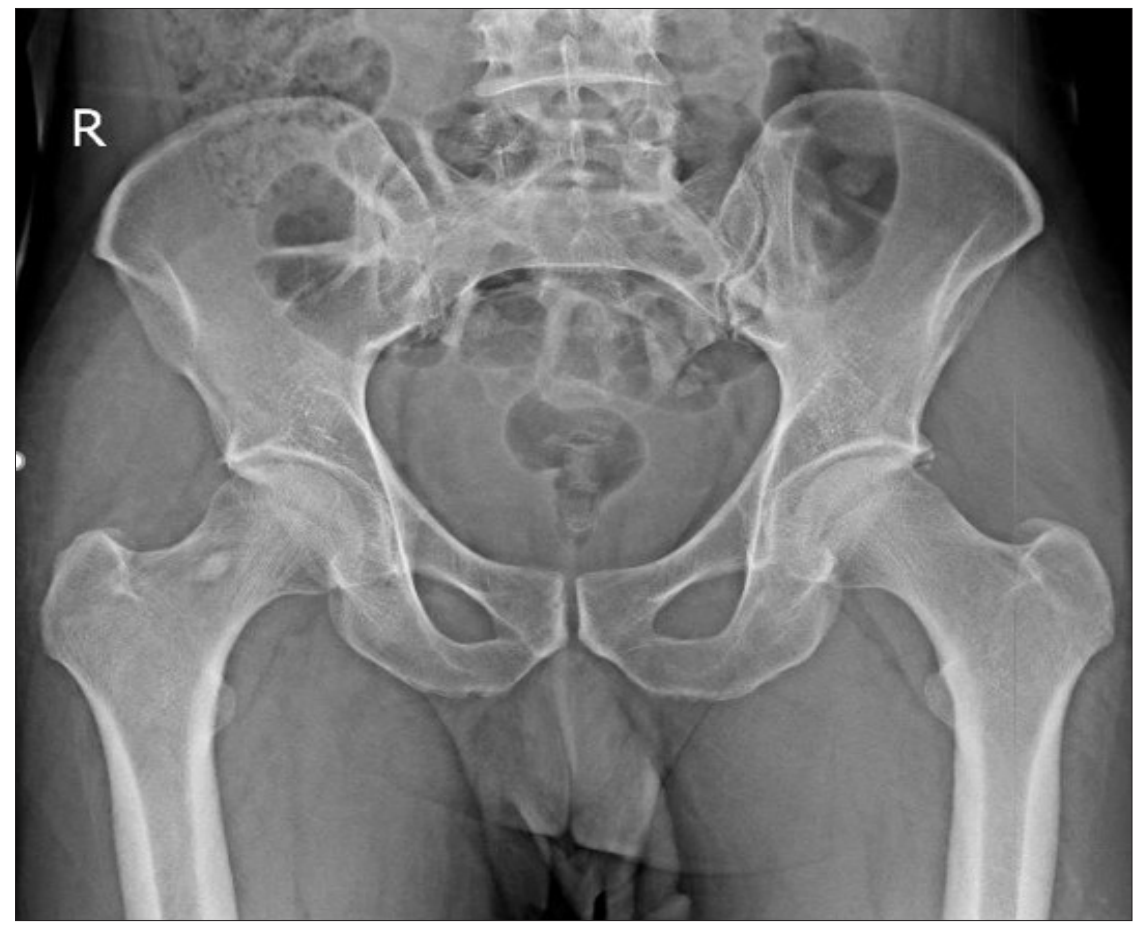

Figure 2: AP pelvic X-ray post reduction. 


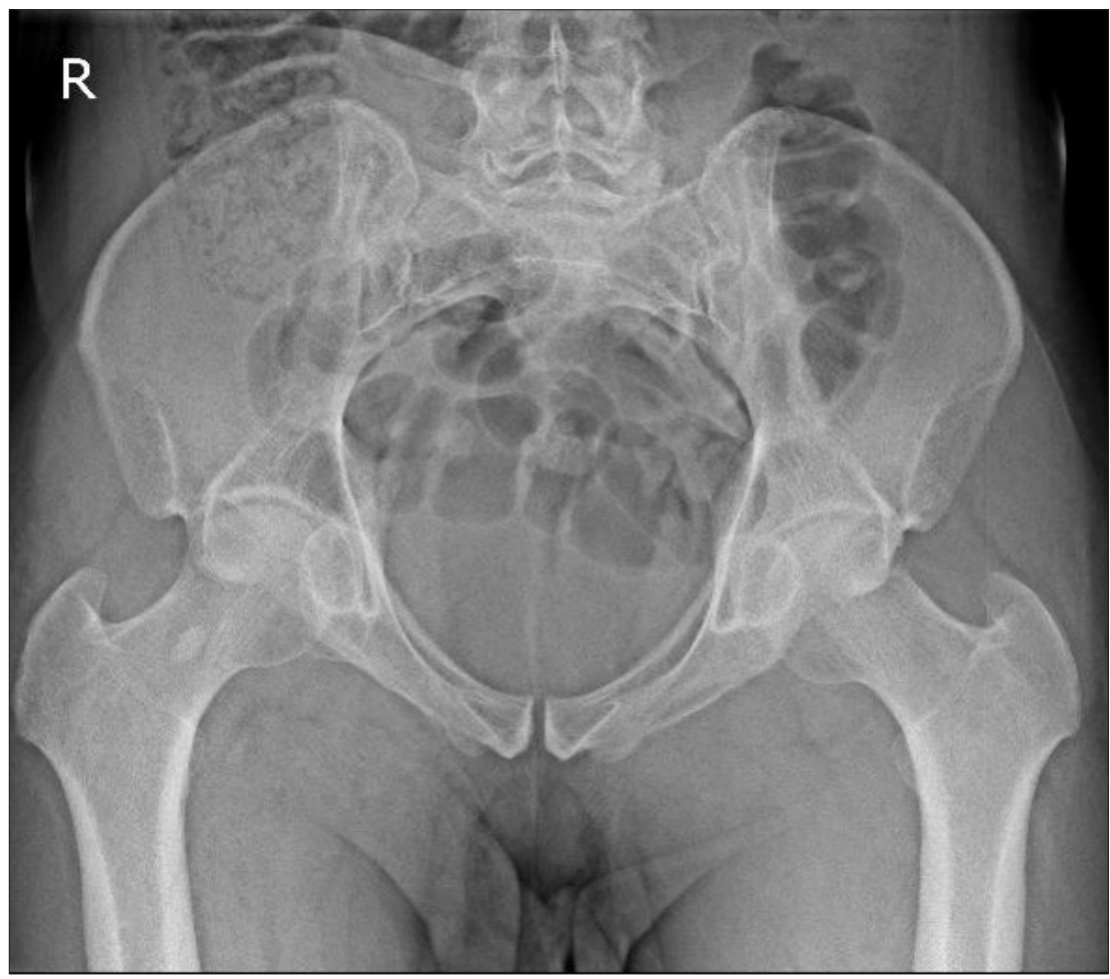

Figure 3: Inlet view pelvic Xray post reduction.

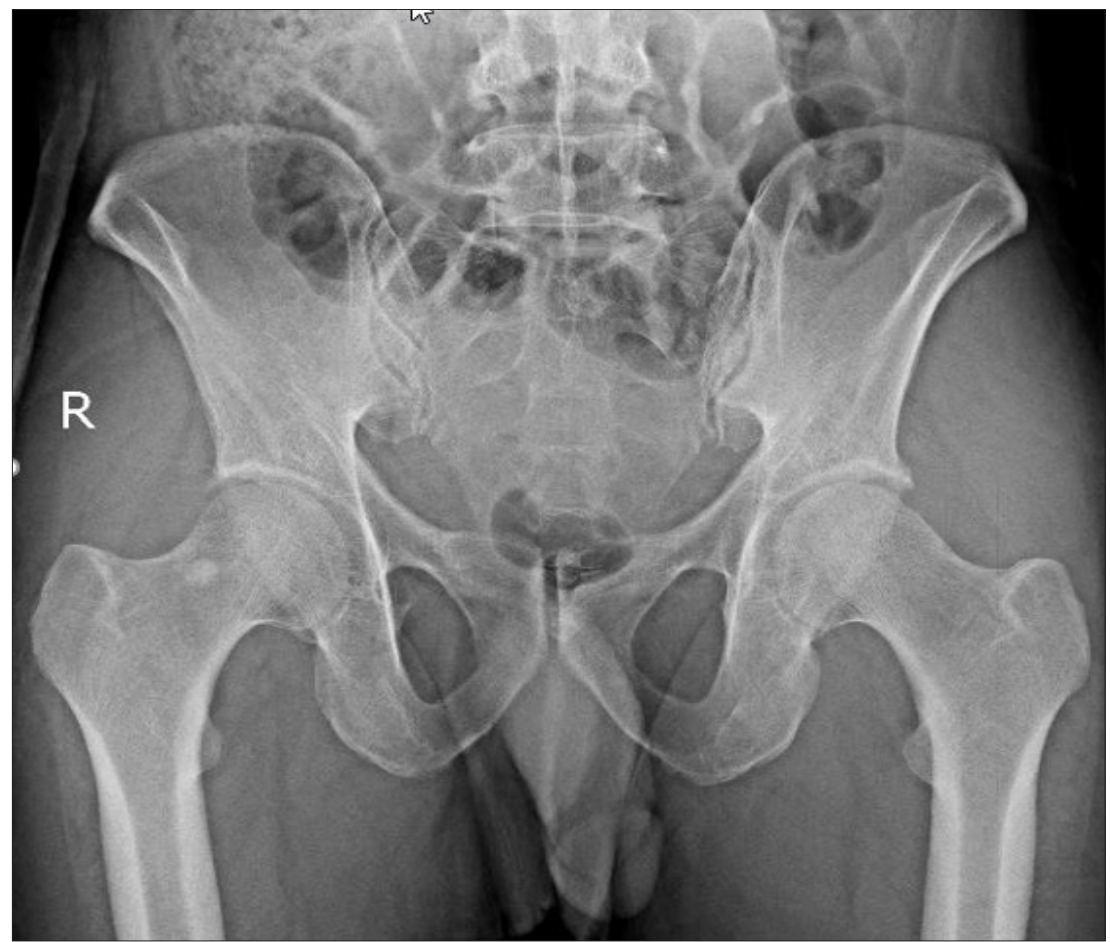

Figure 4: Outlet view pelvic post reduction.

Post reduction X-rays (AP and judet views) were taken showing no fracture (Figures 2-4) or incongruence. $1.5 \mathrm{~mm}$ pelvic CT scan showed no incongruence compared to the contralateral hip joint (Figure 5). Eventually patient was discharged in the following day, ambulating on two crutches with toe touch weight bearing precautions. On the 12 months follow up patient reached the preinjury activity level and Harris hip score [10] measured 95. Medical consent was taken from the patient after clarifying the article (Figure 6). 


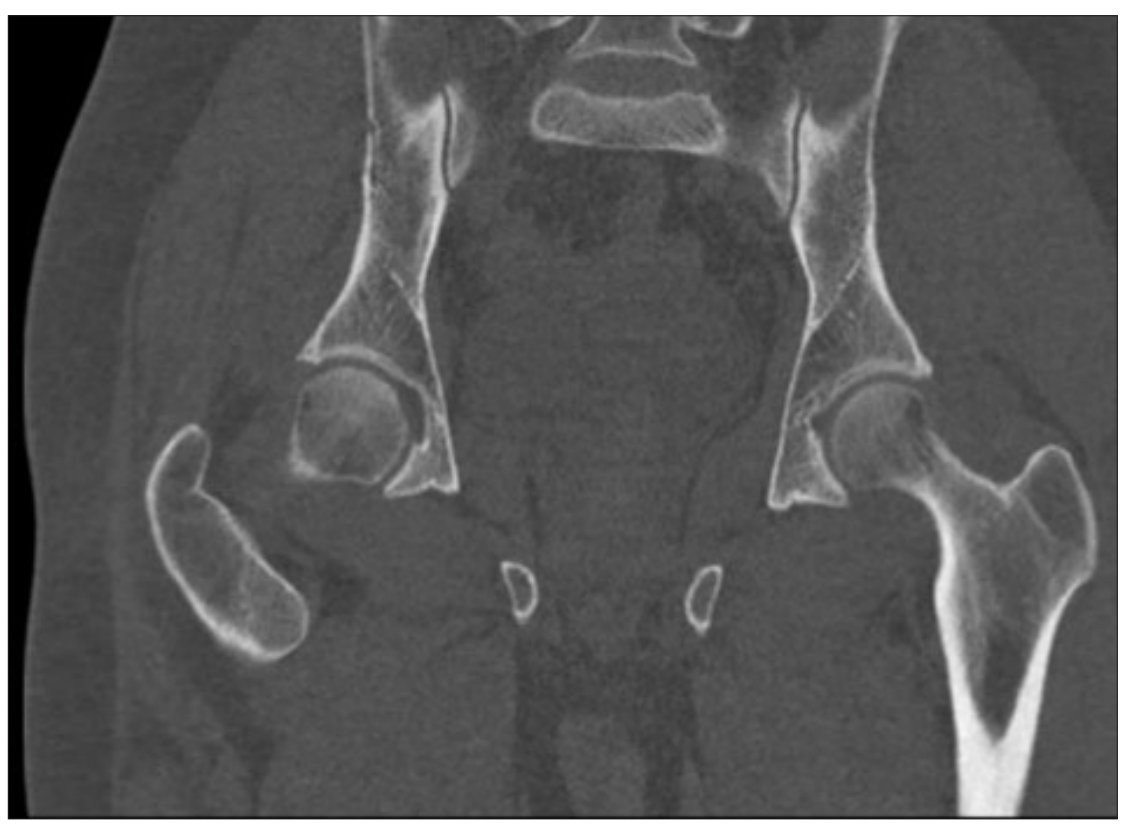

Figure 5: post-reduction pelvic CT scan.

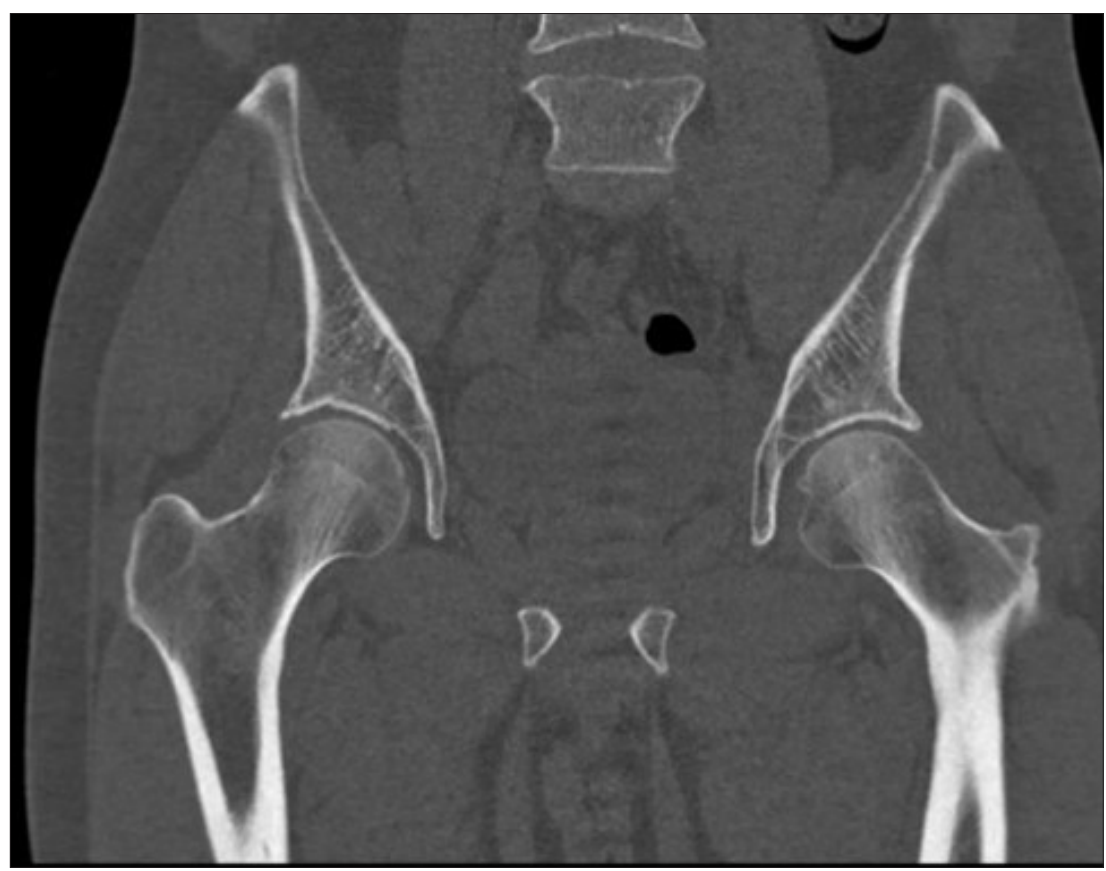

Figure 6: post-reduction pelvic CT scan.

\section{Discussion}

Hip dislocation requires a high energy trauma to overcome the stability of the hip joint [1]. As discussed before true inferior hip dislocations are rare and ligamentous rupture, neurovascular damage or ipsilateral coexisting fractures happen before the disruption of the pelvic harmony. In this article we have reported a single case of inferior hip dislocation (without associated fracture) treated by closed reduction at our ER and ambulated the day after.
Beforehand, it was thought that inferior dislocations are specified to children due to ligamentous laxity, but recent publishes have proved adolescences and adults to be vulnerable as well. Our study is another proof to this claim $[3,9]$. Two different mechanisms are described to cause inferior hip dislocations: In the first, more common, mechanism the femoral head has laid at the same region as the acetabulum. By the second mechanism the femoral head has laid below the acetabulum. As the images of our case clarifies; our case is categorized as the second rarer kind [8]. 
The hint to our discussed case is the pure dislocation and clear post reduction pelvic X-ray and CT scans although following a high energy trauma. Against the discussed inferior hip dislocations in literature our patient had no fracture in the same or contralateral limb or upper extremities. Therefore, the patient was discharged the next day [11,12]; (Figure 7 \& 8).

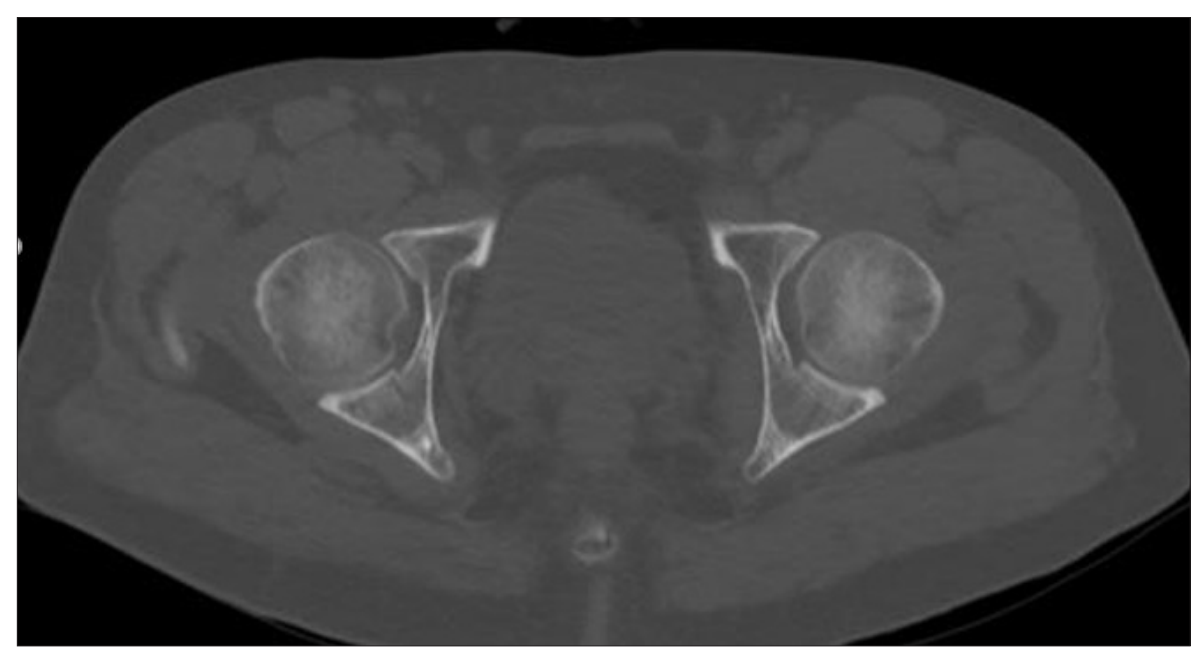

Figure7: post-reduction pelvic CT scan.

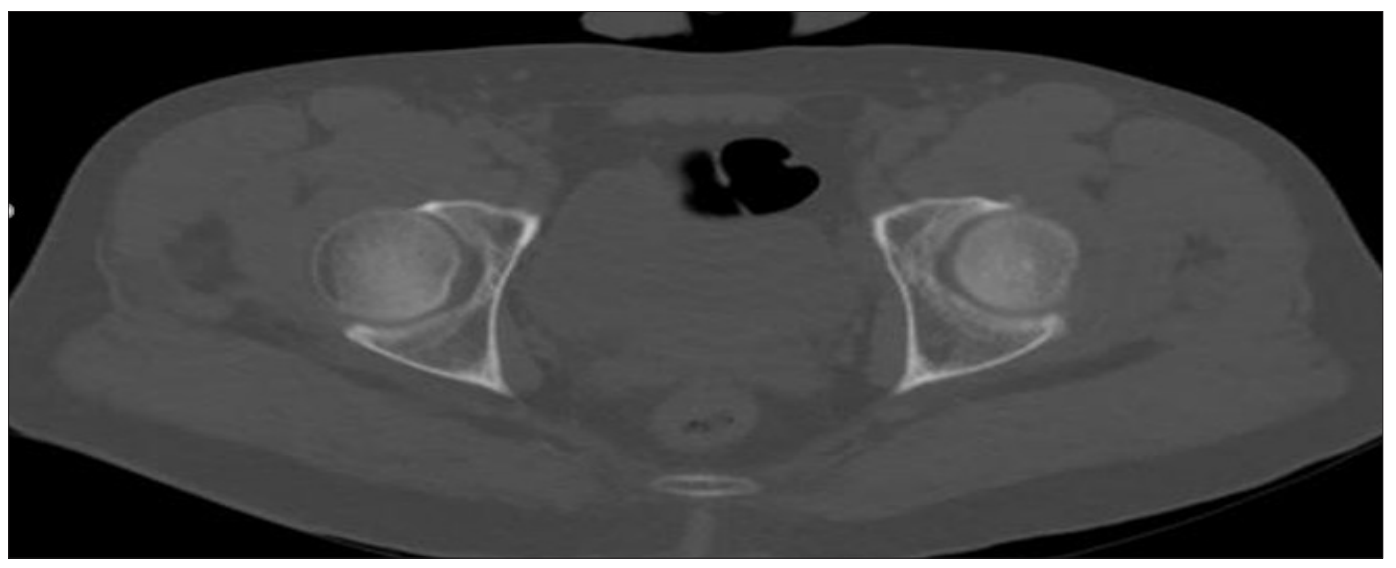

Figure 8: post-reduction pelvic CT scan.

\section{Conclusion}

Inferior hip dislocation is an extremely rare and very few cases have been published in English literature so far. As the high energy accidents are growing in incidence so number of cases to be published is increasing. Through our article the idea that this injury is exclusive to children is once again disproved. The patient undergoes a simple reduction maneuver, considering no co-injury patient is discharged the next day ambulating on crutches. Close reduction gives good results in cases of pure inferior hip dislocation as it happened to our patient.

\section{References}

1. Tekin AÇ, Çabuk H, Büyükkurt CD, Dedeoğlu SS, İmren Y, et al. (2016) Inferior hip dislocation after falling from height: A case report. International Journal of Surgery Case Reports 1(22): 62-65.
2. Ismael S, Vora J, Thomas $P$ (2017) Adult traumatic inferior hip dislocation: Rare case ended with open reduction. Journal of Orthopaedic Case Reports 7(1): 101.

3. Kulambi V, Chaudhari K, Pethapara V (2018) Anterior hip dislocation in young patient obturator (inferior) type: A rare case report. Int J Res Orthop 4(3): 523-526.

4. Beauchesne R, Kruse R, Stanton RP (1994) Inferior dislocation (luxatio erecta) of the hip. Orthopedics 17: 72-75.

5. Lamberti PM, Rabin SI (2003) Open anterior-inferior hip dislocation. J Orthop Trauma 17(1): 65-66.

6. Ferguson KL, Harris VV (2000) Inferior hip dislocation in an adult: does a rare injury now have a common mechanism. Am J Emerg Med 18(1): $117-118$.

7. Brogdon BG, Woolridge DA (1997) Luxatio erecta of the hip: a critical retrospective. Skeletal Radiol 26(9): 548-552. 
8. Hani R, Kharmaz M, Berrada MS (2015) Traumatic obturator dislocation of the hip joint: A case report and review of the literature. Pan African Medical Journal 21(1).

9. Singh R, Sharma SC, Goel T (2006) Traumatic inferior hip dislocation in an adult with ipsilateral trochanteric fracture. Journal of Orthopaedic Trauma 20(3): 220-222.

10. Nilsdotter A, Bremander A (2011) Measures of hip function and symptoms: Harris Hip Score (HHS), Hip Disability and Osteoarthritis
Outcome Score (HOOS), Oxford Hip Score (OHS), Lequesne Index of Severity for Osteoarthritis of the Hip (LISOH), and American Academy of Orthopedic Surgeons (AAOS) hip and knee questionnaire. Arthritis Care \& Research 63(S11): S200-207.

11. Dudkiewicz I, Salai M, Horowitz S, Chechik A (2000) Bilateral asymmetric traumatic dislocation of the hip joints. J Trauma 49(2): 336-338

12. Clegg TE, Roberts CS, Greene JW, Prather BA (2010) Hip dislocationsepidemiology, treatment, and outcomes. Injury 41(4): 329-334. 